\title{
Predictors of survival in older adults hospitalized with COVID-19
}

\author{
Brad Tyson $^{1}\left[\right.$ - Laszlo Erdodi ${ }^{2} \cdot$ Ayman Shahein $^{3} \cdot$ Sharmin Kamrun $^{1} \cdot$ Matthew Eckles $^{1} \cdot$ Pinky Agarwal $^{1}$
}

Received: 9 March 2021 / Accepted: 24 June 2021 / Published online: 3 July 2021

(c) Fondazione Società Italiana di Neurologia 2021

\begin{abstract}
Objective This study was designed to investigate clinical characteristics associated with mortality and predictors of survival in older adults hospitalized with COVID-19 with a focus on neurological comorbidities and presenting neurological manifestations.

Methods We compared clinical characteristics in an age- and gender-matched sample of 75 deceased and 75 recovered patients $\left(M_{\mathrm{Age}}=78\right)$ hospitalized with COVID-19 and developed a logistic regression to predict likelihood of survival.

Results Deceased patients were more like to have dementia, altered mental status (AMS), acute respiratory distress syndrome (ARDS), sepsis, mechanical ventilation, and balance difficulties; higher heart rate, respiratory rate, blood urea nitrogen, creatinine, and absolute neutrophils; lower oxygen saturation and absolute lymphocytes; and shorter length of hospitalization. Logistic regression based on three mortality predictors (ARDS, AMS, and length of hospitalization) correctly predicted $87 \%$ of the outcome ( $89 \%$ sensitivity at $85 \%$ specificity).

Conclusions Dementia and AMS were strong predictors of death in older adults hospitalized with COVID-19. Our findings add to the rapidly growing neurology of COVID-19 literature and underscore the importance of early recognition and the incorporation of a mental status examination into the medical assessment of COVID-19.
\end{abstract}

Keywords COVID-19 $\cdot$ Neurology $\cdot$ Psychiatry $\cdot$ Neuropsychology $\cdot$ Dementia $\cdot$ Delirium

\section{Introduction}

The severe acute respiratory syndrome coronavirus 2 (SARS-CoV-2) and the disease it causes, coronavirus disease 2019 (COVID-19) presents with a range of manifestations such as fever, cough, shortness of breath/difficulty breathing, fatigue, nausea/vomiting, and diarrhea. Multiple medical comorbidities have been linked to severe disease and mortality including cardiovascular diseases, cancer, chronic kidney disease, chronic lung diseases, diabetes mellitus, and obesity. Additional mortality-related factors have included older age and male gender with the overall

Brad Tyson

bradggtyson@gmail.com

1 EvergreenHealth Medical Center, Evergreen Neuroscience Institute, Kirkland, WA, USA

2 Department of Psychology, University of Windsor, Windsor, ON, Canada

3 Department of Clinical Neurosciences, University of Calgary, Calgary, AB, Canada
COVID-19 case-fatality ratio approximately 2.4 times higher among men [1,2].

Neurological manifestations are also common in COVID19, particularly altered mental status (AMS), headache, dizziness, myalgia, hyposmia, hypogeusia, balance difficulties, seizure, and stroke [3-9]. Approximately 35-58\% of hospitalized COVID-19 patients experience neurological manifestations with much higher rates reported in older, more vulnerable samples [8-12]. Neurological manifestations in general, and AMS in particular, are associated with severe disease and death due to COVID-19 [4, 5, 9-11]. Neurological comorbidities have also been associated with severe disease and mortality in COVID-19, particularly dementia and stroke $[1,9,13-15]$.

The current study seeks to contribute to this rapidly accumulating neurology of COVID-19 literature by comparing neurological comorbidities and presenting neurological manifestations in an age- and gender-matched sample of deceased and recovered patients hospitalized with COVID19. We expected baseline dementia and AMS to be significantly related to death due to COVID-19, regardless of the age or gender of our sample. In addition, we aimed to 
identify and develop a simple and user-friendly model of mortality predictors.

\section{Method}

\section{Participants}

Data were collected through a clinical chart review of 512 consecutive patients with SARS-CoV-2 infection seen between February 20, 2020, and July 6, 2020, at EvergreenHealth Medical Center in Washington State, the first hospital with reported cases in the USA. All patients had SARS-CoV-2 infection confirmed by polymerase chain reaction testing of a nasopharyngeal sample. The study was approved by an institutional review board and ethical standards committee. A total of 75 deaths due to COVID19 were identified. The comparison group consisted of the first 75 survivors who matched the age and gender of the deceased sample and were still alive at the time of data analysis. The sample was predominately Caucasian (85\%), evenly male and female, with an average age of 78 . The majority of patients came from assisted living facilities and nursing homes (58\%), the initial epicenter of COVID-19 in our region.

\section{Materials}

Data included demographic characteristics, medical history, presenting symptoms, vital signs and laboratory measures at hospital admission, hospitalization characteristics, and patient outcomes. Chart notes were independently reviewed by a neurologist and neuropsychologist, and data were cross-referenced for accuracy. Core COVID-19 symptoms included fever, shortness of breath, cough, weakness/fatigue, anorexia, diarrhea, and nausea/vomiting. Presenting neurological manifestations included AMS, myalgia, headache, dizziness, balance difficulties, hyposmia, hypogeusia, seizure, and stroke. AMS was defined as acute alterations in mental status, representing a change from baseline, with keywords in chart review including confusion, decreased level of consciousness, impaired consciousness, delirium, encephalopathy, somnolent, obtunded, unresponsive, and/ or coma.

\section{Statistical analysis}

Descriptive statistics $[M, S D$, percentage, likelihood ratios (LR)] were computed where relevant. The statistical significance of between-group differences was determined using independent $t$-tests (two-tailed) and Chi-square. Effect size estimates were expressed in Cohen's $d$ and point-biserial correlation coefficients $\left(r_{\mathrm{pb}}\right)$.
A logistic regression classifier with lasso regularization was used to predict the final clinical outcome of patient recovery versus death. This method was chosen due to the large number of clinical metrics surveyed in our study, given that it offers an objective and systematic framework for modeling a high-dimensional set of predictors without succumbing to overfitting or selection bias. The mathematical details of lasso regularization in logistic regression are discussed in detail elsewhere, but in short, lasso regularization only differs from traditional logistic regression in that the cost function contains an additional term beyond the traditional logistic loss function: a regularization term scaled by a constant lambda which penalizes the addition of new predictors to the model. For predictors that offer no consistent predictive value, lasso regularization aims to set the logistic coefficients of these terms to zero, effectively removing them from the final model. Our implementation used MATLAB's statistics and Machine Learning Toolbox function "lassoglm." We partitioned our data per convention into 80:20 training: test sets. We fit our regression on the training set using tenfold cross-validation with gradient descent over one hundred values of lambda and repeated this process one thousand times to explore which clinical characteristics routinely survived regularization (differing each iteration due to the random initialization of gradient descent and tenfold crossvalidation) suggesting they offer high predictive value. This repetition also allowed the generation of confidence intervals for model performance on the randomly partitioned testing sets. We then created a compact logistic regression classifier from our set of high-yield clinical predictors and evaluated its performance on our testing set. Our results were robust to changes in our classifier's hyperparameters, which were chosen by applying an identical methodology to a synthetic data set mirroring the dimensionality and baseline characteristics of our own.

\section{Results}

\section{Univariate analyses}

Medical comorbidities were nearly universal ( $94 \%$ of total sample) with no significant differences between deceased and recovered patients (Table 1). Cardiovascular diseases were the most common comorbidity ( $81 \%$ of total sample). Neurological comorbidities were present in $56 \%$ of the sample with dementia being most frequent (42\%). The only comorbidity significantly more common in deceased patients was dementia. Patients with dementia were significantly older $(M=84.4)$ than those without dementia $(M=74.1)$ : $t(148)=6.48, p<0.001, d=1.09$ (large effect), $r_{\mathrm{pb}}=0.47$. In addition, dementia was more common in female patients (48.8\%) than male patients $(34.3 \%)$ : $\left[\mathrm{LR}=1.42 ; \chi^{2}(1)=3.21\right.$, 
Table 1 Demographic information and medical history

\begin{tabular}{llll}
\hline Demographic/clinical character- & Discharged & Deceased & $p$-value \\
istics & & & \\
\hline Age & $M=77.6$ & $M=79.3$ & 0.335 \\
& $S D=10.5$ & $S D=11.2$ & \\
Ethnicity & & & \\
Caucasian & $67(89 \%)$ & $60(80 \%)$ & 0.113 \\
Asian/Pacific Islander & $5(7 \%)$ & $10(13 \%)$ & 0.174 \\
Hispanic & $0(0 \%)$ & $4(5 \%)$ & 0.043 \\
African American & $2(1 \%)$ & $0(0 \%)$ & 0.155 \\
Unknown/unavailable & $1(1 \%)$ & $1(1 \%)$ & 1.00 \\
Medical comorbidities & $72(96 \%)$ & $69(92 \%)$ & 0.302 \\
Cardiovascular disease & $63(84 \%)$ & $58(77 \%)$ & 0.301 \\
Diabetes & $25(33 \%)$ & $26(35 \%)$ & 0.863 \\
Chronic kidney disease & $20(27 \%)$ & $18(24 \%)$ & 0.707 \\
Obesity & $18(24 \%)$ & $13(18 \%)$ & 0.333 \\
Chronic lung disease & $14(19 \%)$ & $16(21 \%)$ & 0.683 \\
Cancer & $15(84 \%)$ & $15(84 \%)$ & 0.301 \\
Obstructive sleep apnea & $12(16 \%)$ & $15(20 \%)$ & 0.524 \\
Asthma & $5(7 \%)$ & $8(11 \%)$ & 0.384 \\
Neurologic comorbidities & $37(49 \%)$ & $47(63 \%)$ & 0.119 \\
Dementia & $25(33 \%)$ & $38(50 \%)$ & 0.032 \\
Stroke & $11(15 \%)$ & $15(20 \%)$ & 0.388 \\
Brain tumor & $2(3 \%)$ & $5(7 \%)$ & 0.246 \\
Seizure disorder & $3(4 \%)$ & $5(7 \%)$ & 0.467 \\
Parkinson's disease & $2(3 \%)$ & $2(3 \%)$ & 1.00 \\
Multiple sclerosis & $0(0 \%)$ & $2(3 \%)$ & 0.155 \\
Psychiatric comorbidities & $41(55 \%)$ & $37(49 \%)$ & 0.513 \\
Depression & $30(40 \%)$ & $24(32 \%)$ & 0.307 \\
Anxiety & $14(19 \%)$ & $17(23 \%)$ & 0.545 \\
Insomnia & $9(12 \%)$ & $7(9 \%)$ & 0.597 \\
Bipolar disorder & $3(4 \%)$ & $5(7 \%)$ & 0.467 \\
Schizophrenia & $1(1 \%)$ & $5(7 \%)$ & 0.096 \\
\hline
\end{tabular}

$p=0.073]$. Psychiatric conditions were present in half the sample $(52 \%)$ with depression being most frequent $(36 \%)$. There was no difference in psychiatric comorbidity between deceased and recovered patients.

Fever, cough, and shortness of breath were the most common symptoms reported in both groups (Table 2). AMS and balance difficulties were the only symptoms significantly more frequent in deceased patients. All eight patients with coma at admission died. Further, all three patients with seizures died, including one patient who presented with non-convulsive status epilepticus at admission. Of the four patients with ischemic strokes, three died. Recovered patients were more likely to have diarrhea and hyposmia or hypogeusia.

On admission vital signs, deceased patients had higher heart rate (large effect), higher respiratory rate (medium effect), and lower oxygen saturation (medium effect). On
Table 2 Presenting symptoms

\begin{tabular}{llll}
\hline Core symptoms & Discharged & Deceased & $p$-value \\
\hline Fever & $47(63 \%)$ & $54(72 \%)$ & 0.223 \\
Shortness of breath & $41(55 \%)$ & $53(71 \%)$ & 0.058 \\
Cough & $55(73 \%)$ & $44(59 \%)$ & 0.058 \\
Weakness/fatigue & $37(49 \%)$ & $38(51 \%)$ & 0.870 \\
Anorexia & $23(31 \%)$ & $24(32 \%)$ & 0.860 \\
Diarrhea & $24(32 \%)$ & $13(17 \%)$ & 0.037 \\
Nausea/vomiting & $7(9 \%)$ & $5(7 \%)$ & 0.547 \\
Neurologic symptoms & $53(71 \%)$ & $62(81 \%)$ & 0.082 \\
Altered mental status & $34(45 \%)$ & $50(67 \%)$ & 0.008 \\
Coma at admission & $0(0 \%)$ & $8(11 \%)$ & 0.004 \\
Dizziness & $11(15 \%)$ & $9(12 \%)$ & 0.631 \\
Balance difficulties & $5(7 \%)$ & $14(19 \%)$ & 0.027 \\
Myalgia & $7(9 \%)$ & $12(16 \%)$ & 0.220 \\
Headache & $10(13 \%)$ & $5(7 \%)$ & 0.174 \\
Hyposmia & $2(3 \%)$ & $0(0 \%)$ & 0.155 \\
Hypogeusia & $5(7 \%)$ & $0(0 \%)$ & 0.023 \\
Stroke & $1(1 \%)$ & $3(4 \%)$ & 0.311 \\
Seizure & $0(0 \%)$ & $3(4 \%)$ & 0.080 \\
\hline
\end{tabular}

laboratory serology, deceased patients showed lower absolute lymphocytes and higher blood urea nitrogen, creatinine, and absolute neutrophils (medium effects; Table 3).

Complications and hospitalization characteristics observed more frequently in deceased patients included acute respiratory distress syndrome (ARDS), sepsis, mechanical ventilation, and admission to critical care. Higher survival rate was observed in patients treated with hydroxychloroquine $\left[\mathrm{LR}=1.73 ; \chi^{2}(1)=11.0, p=0.001\right]$ or remdesivir $\left[\mathrm{LR}=1.67 ; \chi^{2}(1)=3.86, p=0.050\right]$. Recovered patients had a significantly longer length of hospitalization $(p<0.001)$ with deceased patients averaging just over a week in the hospital $(M=7.6$ days, $S D=6.3)$ and recovered patients averaging more than 3 weeks inpatient $(M=23.4$ days, $S D=18.9)$.

\section{Multivariate analyses}

Patient outcomes of death versus recovery were classified using logistic regression with lasso regularization. Of 115 variables available in the analysis, our regularized models favored the inclusion of three clinical characteristics: ARDS, AMS, and length of hospitalization. ARDS and AMS were binary predictors (set to 0 if absent, 1 if present), whereas the length of hospitalization took integer values for duration in days. Building a logistic regression with these three clinical predictors improved on the performance of a naive logistic regression (using all 115 predictors) when applied to our testing set by avoiding overfitting, with the added benefit of improving model simplicity. Our final classifier 
Table 3 Admission vital signs and laboratory measures

\begin{tabular}{|c|c|c|c|c|c|c|c|c|}
\hline \multirow[b]{3}{*}{ Vital signs } & \multicolumn{4}{|c|}{ Clinical outcome } & \multirow[b]{3}{*}{$t$} & \multirow[b]{3}{*}{$p$} & \multirow[b]{3}{*}{$d$} & \multirow[b]{3}{*}{$\sigma_{1}$ vs $\sigma_{2}$} \\
\hline & \multicolumn{2}{|c|}{ Survived } & \multicolumn{2}{|c|}{ Deceased } & & & & \\
\hline & M & $S D$ & M & $S D$ & & & & \\
\hline Temperature $\left({ }^{\circ} \mathrm{C}\right)$ & 37.3 & 0.92 & 37.4 & 0.83 & 0.59 & 0.558 & - & 0.426 \\
\hline Respiratory rate & 21.3 & 5.8 & 25.0 & 11.2 & 2.48 & 0.015 & 0.41 & 0.013 \\
\hline Oxygen saturation & 94.5 & 4.84 & 91.2 & 7.29 & 3.26 & 0.001 & 0.53 & 0.003 \\
\hline Heart rate & 82.1 & 16.9 & 96.0 & 23.5 & 4.14 & $<0.001$ & 0.68 & 0.016 \\
\hline BP, systolic & 134.5 & 22.8 & 127.0 & 23.9 & 1.95 & 0.053 & 0.32 & 0.488 \\
\hline $\mathrm{BP}$, diastolic & 71.8 & 12.5 & 73.8 & 17.0 & 0.79 & 0.429 & - & 0.151 \\
\hline Laboratory measures & M & $S D$ & M & $S D$ & $t$ & $p$ & $d$ & $\sigma_{1}$ vs $\sigma_{2}$ \\
\hline White blood cell, /mL & 7.9 & 5.1 & 8.9 & 4.8 & 1.14 & 0.255 & - & 0.328 \\
\hline Hemoglobin, g/DL & 12.5 & 2.2 & 11.9 & 2.2 & 1.60 & 0.111 & - & 0.930 \\
\hline Hematocrit, \% & 38.6 & 6.2 & 37.1 & 6.1 & 1.39 & 0.167 & - & 0.873 \\
\hline Absolute platelet, $103 / \mathrm{mL}$ & 224.7 & 112.3 & 207.5 & 91.2 & 1.01 & 0.316 & - & 0.247 \\
\hline Sodium, mmol/L & 137 & 5.9 & 137.6 & 7.4 & 0.50 & 0.617 & - & 0.085 \\
\hline Potassium, mmol/L & 4.1 & 0.6 & 4.0 & 0.6 & 0.28 & 0.783 & - & 0.603 \\
\hline Chloride, $\mathrm{mEq} / \mathrm{L}$ & 98.3 & 6.1 & 98.6 & 6.9 & 0.23 & 0.821 & - & 0.386 \\
\hline Carbon dioxide, $\mathrm{mEq} / \mathrm{L}$ & 24.3 & 3.3 & 24.1 & 4.7 & 0.28 & 0.780 & - & 0.185 \\
\hline Blood urea nitrogen, $\mathrm{mmol} / \mathrm{L}$ & 22.7 & 12.5 & 32.0 & 19.1 & 3.41 & 0.001 & 0.58 & 0.003 \\
\hline Creatinine, mg/dL & 1.1 & 0.6 & 1.6 & 1.7 & 2.24 & 0.027 & 0.39 & 0.008 \\
\hline Glucose, $\mathrm{mmol} / \mathrm{L}$ & 134 & 54.0 & 144.2 & 53.6 & 1.10 & 0.275 & - & 0.851 \\
\hline Alanine aminotransferase, $\mathrm{U} / \mathrm{L}$ & 35.1 & 38.7 & 31.5 & 33.1 & 0.58 & 0.565 & - & 0.362 \\
\hline Aspartate aminotransferase, U/L & 46.1 & 51.1 & 53.7 & 54.1 & 0.82 & 0.412 & - & 0.465 \\
\hline Lactic acid, mmol/L & 1.7 & 0.8 & 2.1 & 1.5 & 1.49 & 0.140 & - & 0.026 \\
\hline C-reactive protein, mg/DL & 9.4 & 10.0 & 10.8 & 6.8 & 0.62 & 0.537 & - & 0.133 \\
\hline Creatine kinase, U/L & 210.4 & 445.2 & 496.5 & 1221.6 & 1.36 & 0.179 & - & 0.057 \\
\hline Lymphocyte number, 10E3/uL & 1.2 & 0.8 & 0.9 & 0.7 & 2.22 & 0.029 & 0.40 & 0.190 \\
\hline Neutrophil number, 10E3/uL & 5.6 & 3.7 & 7.3 & 4.4 & 2.28 & 0.024 & 0.42 & 0.088 \\
\hline
\end{tabular}

Note: $B P$, blood pressure; $\sigma_{1} v s \sigma_{2}, p$-value associated with Levene's test of homogeneity of variance assigned logistic coefficients of 3.70 to ARDS, 1.97 to AMS, and -0.17 to length of hospitalization, with the logistic constant set to -0.86 . This suggests that ARDS, AMS, and short length of hospitalization were associated with poor outcomes. This model performed on our testing sets with a sensitivity of $89 \%$ ( $\mathrm{SE}=6 \%$ ), a specificity of $85 \%(\mathrm{SE}=8 \%)$, and a total classification accuracy of $87 \%(\mathrm{SE}=6 \%)$.

\section{Discussion}

Consistent with an increasing body of literature, dementia and AMS were significant predictors of mortality in older adults hospitalized with COVID-19 [1, 4, 5, 9-15]. Dementia was the only comorbidity associated with death in our sample. Contrary to other studies [16], psychiatric comorbidities were not associated with death in our sample, suggesting this variable is prone to sample-specific fluctuations. Age and gender were unrelated to outcome in our study, contradicting previous reports $[1,2]$. However, these demographic variables were correlated with dementia, suggesting the predictive power of age and gender in previous studies was confounded by another variable, such as dementia.

AMS and balance difficulties were the only presenting symptoms more common in deceased patients. Survivors were significantly more likely to have diarrhea, a finding consistent with research linking digestive symptoms, specifically diarrhea, with milder disease and longer delays before viral clearance [17]. No deceased patients presented with hypogeusia or hyposmia, which is also consistent with these symptoms being more common in large-scale studies comprised of younger patients with milder disease [8, $15,16,18]$. Further, AMS and dementia likely masked the frequency of these symptoms. The use of hydroxychloroquine and/or remdesivir extended the length of hospitalization for survivors, but we think this was less likely related to the effectiveness of these drugs and more likely because they were used on healthier patients with higher chances of recovery.

Our analysis culminated in a compact and efficient logistic regression based on three parameters readily available to medical professionals: ARDS, AMS, and length of 
hospitalization. The model accurately predicted outcomes $87 \%$ of the time, providing a balance between sensitivity (89\%) and specificity (85\%). Although ARDS and AMS as predictors of mortality make intuitive sense, one might think that the length of hospitalization would be a negative predictor of survival. In this view, the healthiest patients are discharged quickly, and the sickest patients endure extended hospitalizations ending in death. Contrary to expectation, patients with longer hospitalizations had more favorable outcomes. There are several potential explanations for this. Our data was gathered at the onset of the pandemic in the USA, when nursing homes and assisted living facilities struggled to contain the virus. Only the most severely ill patients were sent to the hospital, many with do not resuscitate orders, with palliative care as the main goal. Combined with a lack of information regarding early recognition of COVID-19 and its diverse manifestations in older adults, this patient stream likely experienced delays from infection to hospitalization and, as a result, shorter hospitalizations due to more severe disease, rapid deterioration, and death. Given these circumstances, if patients received more immediate medical attention and/or could survive the most critical phase of illness leading up to admission, they were more likely to be hospitalized longer but also more likely to recover. Our model is completely linear, meaning that it can't see a single predictor as being good and bad in different contexts: it couldn't discern if a short length of hospitalization in some cases was a positive (early discharge) or negative (rapid deterioration to death) predictor of survival. The model viewed length of hospitalization unilaterally as a marker of positive outcomes. Thus, longer hospitalization was always associated with a more favorable outcome in our sample.

There were several limitations of the current study worth mentioning. Retrospective chart reviews can result in underassessment and underdocumentation of mild or secondary symptoms. The severity of AMS was ostensibly associated with mortality in our study, as evidenced by all eight of our coma patients dying; however, it would be useful to know the mortality rate at lower ends of the AMS spectrum. To that end, future research would benefit from prospective studies using standardized mental status examinations or other brief cognitive screeners to predict shortand long-term outcomes. The sample size was modest and restricted in terms of racial and ethnic diversity compared to other studies in this area. Our sample was older with multiple medical comorbidities including high rates of dementia, many of whom were residing in nursing homes or assisted living facilities and gathered during the onset of the pandemic from a single hospital in the Northwest United States. These factors limit the generalizability of our findings to younger healthier patients, ethnically diverse patients, community-dwelling older adults, other geographic locations, and later stages of the pandemic. As such, our predictive model needs independent cross-validation to determine its clinical utility in different settings. Finally, our study would also benefit from data on additional hospitalization characteristics associated with death and survival, such as changing laboratory markers over the course of disease progression and results of other diagnostic tests.

In sum, our findings add to the rapidly growing neurology of COVID-19 literature and underscore the importance of early identification and the incorporation of a mental status examination in the medical assessment of COVID-19. As we move into the second year of this pandemic, data on long-term neurological outcomes will quickly accumulate. With survivors increasingly seeking healthcare services in the coming years, clinicians need to consider the high potential of neurological and psychiatric complications due to COVID-19. Ongoing research in recovered patients should include follow-up neurological and neuroimaging studies to record potential long-term neurological complications and neuropsychological evaluation to document the extent of any persisting cognitive, emotional, and behavioral sequelae.

Availability of data and material Anonymized data will be shared upon reasonable request for a study that has been approved by a local ethical committee.

\section{Declarations}

Ethical approval Study is in compliance with ethical standards.

Conflict of interest The authors declare no competing interests.

Informed consent The study was approved by an institional review board and local ethics committee.

\section{References}

1. Centers for Disease Control (2021) People at increased risk for severe illness. https://www.cdc.gov/coronavirus/2019-ncov/needextra-precautions/index.html. Accessed 30 Jan 302021

2. Griffith D, Sharma G, Holliday C et al (2020) Men and COVID19: a biopsychosocial approach to understanding sex differences in mortality and recommendations for practice and policy interventions. Prev Chronic Dis 17:1-8

3. Agarwal P, Ray S, Madan A, Tyson B (2020) Neurological manifestations in 404 COVID-19 patients in Washington State. J Neurol.https://doi.org/10.1007/s00415-020-10087-z

4. Chen T, Wu D, Chen H et al (2020) Clinical characteristics of 113 deceased patients with coronavirus disease 2019: retrospective study. BMJ 368:1-12

5. Mao L, Jin H, Wang M et al (2020) Neurological manifestations of hospitalized patients with COVID-19 in Wuhan, China. JAMA Neurol 77:683-690

6. Favas TT, Dev P, Chaurasia RN et al (2020) Neurological manifestations of COVID-19: a systematic review and meta-analysis of proportions. Neurol Sci 21:1-34 
7. Niazkar HR, Zibaee B, Nasimi A, Bahri N (2020) The neurological manifestations of COVID-19: a review article. Neurol Sci 41:1667-1671

8. Karadaş O, Ozturk B, Sonkaya AR (2020) A prospective clinical study of detailed neurological manifestations in patients with COVID-19. Neurol Sci 41:1991-1995

9. Tyson B, Erdodi L, Ray S, Agarwal P (2020) Altered mental status in 71 deaths due to COVID-19. Int J Neurosci 30:1-4

10. Helms J, Kremer S, Merdji H et al (2020) Neurologic features in severe SARS-CoV-2 infection. N Eng J Med 382:2268-2270

11. Romero-Sánchez CM, Díaz-Maroto I, Fernandez-Diaz et al (2020) Neurologic manifestations in hospitalized patients with COVID19: the ALBACOVID Registry. Neurology. https://doi.org/10. 1212/WNL.0000000000009937

12. Vacchiano V, Riguzzi P, Volpi L et al (2020) Early neurological manifestations of hospitalized COVID-19 patients. Neurol Sci 41:2029-2031

13. García-Azorín D, Martínez-Pías E, Trigo J et al (2020) Neurological comorbidity is a predictor of death in COVID-19 disease: a cohort study on 576 patients. Front Neurol 11:1-8

14. Eskandar E, Altschul D, Ramos R et al (2020) Neurologic syndromes predict higher in-hospital mortality in COVID-19. Neurology.https://doi.org/10.1212/WNL.0000000000011356
15. Hwang J, Kim J, Park J, Chang M, Park D (2020) Neurological diseases as mortality predictive factors for patients with COVID19: a retrospective cohort study. Neurol Sci 41:2317-2324

16. Li L, Li F, Fortunati F, Krystal J (2020) Association of a prior psychiatric diagnosis with mortality among hospitalized patients with coronavirus disease 2019 (COVID-19) infection. JAMA 3:e2023282-e2023282

17. Han C, Duan C, Zhang S et al (2020) Digestive symptoms in COVID-19 patients with mild disease severity: clinical presentation, stool viral RNA testing, and outcomes. Am J Gastroenterol 115:916-923

18. Lechien J, Chiesa-Estomba C, Beckers E, et al (2021) Prevalence and 6-month recovery of olfactory dysfunction: a multicentre study of 1363 COVID-19 patients. J Intern Med.https://doi.org/ 10.1111/joim. 13209

Publisher's note Springer Nature remains neutral with regard to jurisdictional claims in published maps and institutional affiliations. 